\title{
Atténuation d'une onde à la surface d'un conducteur entouré de diélectriques
}

\author{
G. Zepp, A. Wick et G. Fabre \\ Physique des ondes, U.E.R. des Sciences, Université de Clermont II, B.P. 45, 63170 Aubière, France
}

(Reçu le 1 ${ }^{\mathrm{er}}$ décembre 1980, accepté le 2 mars 1981)

\begin{abstract}
Résumé. - On montre qu'il est possible d'obtenir simultanément un faible coefficient d'atténuation et une bonne concentration de l'énergie pour une onde électromagnétique T.M. se propageant à la surface d'un conducteur ou d'un supraconducteur cylindrique entouré de diélectriques.
\end{abstract}

\begin{abstract}
We are studying the T.M. electromagnetic wave propagation on the surface of a cylindrical conductor or superconductor coated with dielectrics. The compatibility between a low attenuation factor and a good localization of the power propagated is shown.
\end{abstract}

Introduction. - On connaît l'intérêt qu'il y a, pour une onde électromagnétique T.M. se propageant au voisinage de la surface d'un conducteur cylindrique, à gainer celui-ci d'une première couche très mince de diélectrique. On obtient ainsi un très faible coefficient d'atténuation [1-6] mais au détriment d'une bonne localisation de l'énergie autour du conducteur; l'énergie est en effet mieux concentrée au voisinage immédiat du conducteur si l'épaisseur de la couche de diélectrique n'est pas trop faible devant le rayon du conducteur. La méthode que nous développons conduit à des expressions générales analytiques plus précises que celles habituellement admises pour les grandeurs intéressantes (coefficient d'atténuation, déphasage, ...). Elle permet une généralisation au cas des supraconducteurs et elle montre qu'il est possible d'obtenir des valeurs faibles du coefficient d'atténuation pour des épaisseurs notables de la première couche de diélectrique.

Nous étudions en détail le cas d'un métal conducteur ou supraconducteur de forme cylindrique à section circulaire entouré de deux diélectriques.

On s'intéresse à la propagation le long d'un cylindre conducteur ou supraconducteur, infiniment long, d'axe $z^{\prime} z$, de rayon $a$, le premier diélectrique, de constante diélectrique $\varepsilon$, occupant l'espace compris entre les cylindres de rayons $a$ et $a^{\prime}$ et le deuxième diélectrique, de permittivité $\varepsilon^{\prime}$, occupant tout le reste de l'espace. Par hypothèse $|\varepsilon|>\left|\varepsilon^{\prime}\right|$. Dans un système de coordonnées cylindriques $(r, \varphi, z)$, les parties radiales des champs $E_{z}$ et $H_{\varphi}$ dans le premier diélectrique sont respectivement des combinaisons linéaires de $J_{0}$ et $Y_{0}$ et de $J_{1}$ et $Y_{1}$. En exprimant la continuité des composantes tangentielles des champs $E_{z}$ et $H_{\varphi}$ il vient :

pour

et pour

$$
r=a \quad \frac{E_{z}}{H_{\varphi}} \simeq Z_{\mathrm{s}}
$$

d'où :

$$
r=a^{\prime} \quad \frac{E_{z}}{H_{\varphi}} \simeq \omega \mu_{0} \frac{\kappa^{\prime}}{k^{\prime 2}} \frac{H_{0}^{(1)}\left(i \kappa^{\prime} a^{\prime}\right)}{H_{1}^{(1)}\left(i \kappa^{\prime} a^{\prime}\right)}
$$

$\frac{\omega}{i} \mu_{0} \frac{\chi}{k^{2}} \frac{\left\{J_{0}\left(\chi a^{\prime}\right) Y_{0}(\chi a)-Y_{0}\left(\chi a^{\prime}\right) J_{0}(\chi a)+\Delta_{0}\left(Y_{0}\left(\chi a^{\prime}\right) J_{1}(\chi a)-J_{0}\left(\chi a^{\prime}\right) Y_{1}(\chi a)\right)\right\}}{J_{1}\left(\chi a^{\prime}\right) Y_{0}(\chi a)-Y_{1}\left(\chi a^{\prime}\right) J_{0}(\chi a)+\Delta_{0}\left[Y_{1}\left(\chi a^{\prime}\right) J_{1}(\chi a)-J_{1}\left(\chi a^{\prime}\right) Y_{1}(\chi a)\right]} \simeq \omega \mu_{0} \frac{\kappa^{\prime}}{k^{\prime 2}} \frac{H_{0}^{(1)}\left(i \kappa^{\prime} a^{\prime}\right)}{H_{1}^{(1)}\left(i \kappa^{\prime} a^{\prime}\right)}$

avec

$$
\begin{array}{ll}
\chi^{2} & =k^{2}-\gamma^{2}, \\
\kappa^{\prime 2} & =\gamma^{2}-k^{\prime 2}, \\
\Delta_{0} & =\frac{i k^{2} Z_{\mathrm{s}}}{\mu_{0} \omega \chi}
\end{array}
$$


où : $\omega$ est la pulsation de l'onde de fréquence $f, \gamma$ la constante de propagation et

$$
Z_{\mathrm{s}}=R_{\mathrm{s}}+i X_{\mathrm{s}}
$$

l'impédance de surface du métal, $J, Y$ et $H$ étant respectivement les fonctions de Bessel, de Neumann et de Hankel. Définissons :

$$
\begin{aligned}
& x^{\prime}=\chi a^{\prime} \\
& y^{\prime}=\kappa^{\prime} a^{\prime} .
\end{aligned}
$$

Compte tenu de $\left|x^{\prime}\right| \ll 1$ et $\left|y^{\prime}\right| \ll 1$ lorsque $a^{\prime}$ est nettement plus petit que la longueur d'onde $\lambda$, de (1) on déduit :

$$
\begin{aligned}
x^{\prime 2} \ln \frac{a^{\prime}}{a}\{1 & \left.+\left(\frac{x^{\prime}}{2}\right)^{2} F\left(\frac{a^{\prime}}{a}\right)-\Delta_{0}\left(\frac{x^{\prime}}{2} G\left(\frac{a^{\prime}}{a}\right)+\frac{1}{x^{\prime}} \frac{a^{\prime}}{a} \frac{1}{\ln \frac{a^{\prime}}{a}}\right)\right\} \simeq \\
& \simeq-\frac{|\varepsilon|}{\left|\varepsilon^{\prime}\right|} \mathrm{e}^{-i\left(\varphi-\varphi^{\prime}\right)} y^{\prime 2} \ln \left(0,89 y^{\prime}\right)\left\{1+2\left(\frac{y^{\prime}}{2}\right)^{2}-2\left(\frac{y^{\prime}}{2}\right)^{2} \ln \left(0,89 y^{\prime}\right)-\frac{\left(\frac{y^{\prime}}{2}\right)^{2}}{\ln \left(0,89 y^{\prime}\right)}\right\} .
\end{aligned}
$$

Dans cette expression $\varphi$ et $\varphi^{\prime}$ représentent les angles de pertes des deux diélectriques

et

$$
\left(\varepsilon=|\varepsilon| \mathrm{e}^{-i \varphi}, \quad \varepsilon^{\prime}=\left|\varepsilon^{\prime}\right| \mathrm{e}^{-i \varphi^{\prime}}\right)
$$

$$
\begin{aligned}
& F\left(\frac{a^{\prime}}{a}\right)=\frac{1-\left(\frac{a}{a^{\prime}}\right)^{2}}{\ln \frac{a^{\prime}}{a}}-2\left(1-\ln \frac{a^{\prime}}{a}\right) \\
& G\left(\frac{a^{\prime}}{a}\right)=\frac{a^{\prime}}{a}+\frac{1}{2} \frac{1}{\ln \frac{a^{\prime}}{a}}\left(\frac{a}{a^{\prime}}-\frac{a^{\prime}}{a}\right) .
\end{aligned}
$$

Par ailleurs, d'après (2) et (5) :

$$
x^{\prime 2}+y^{\prime 2}=\mu_{0} \omega^{2}\left(|\varepsilon| \mathrm{e}^{-i \varphi}-\left|\varepsilon^{\prime}\right| \mathrm{e}^{-i \varphi^{\prime}}\right) a^{\prime 2}
$$

De (6) et (8) on peut déduire $x^{\prime}$ et $y^{\prime}$ et partant la constante de propagation $\gamma$ d'après (2).

On pose :

$$
\gamma=\gamma_{0}+\delta \gamma
$$

avec $|\delta \gamma| \ll \gamma_{0}, \gamma_{0}$ étant la constante de propagation en l'absence de pertes. En séparant partie réelle et partie imaginaire de $\delta \gamma$

$$
\delta \gamma=\delta \gamma_{0}-i \alpha
$$

on obtient $\delta \gamma_{0}$ et le coefficient d'atténuation $\alpha$.

$$
\begin{gathered}
\delta_{\gamma_{0}} \simeq \frac{1}{\gamma_{0} a^{\prime 2}} \frac{\left[|\varepsilon| \omega a^{\prime} x_{0}^{\prime} \ln \frac{a^{\prime}}{a}\left(\frac{G}{2} x_{0}^{\prime}+\frac{1}{x_{0}^{\prime}} \frac{a^{\prime}}{a} \frac{1}{\left.\ln \frac{a^{\prime}}{a}\right)} X_{\mathrm{s}}\right]\right.}{\left[2 \ln \frac{a^{\prime}}{a}\left(\frac{F}{2} x_{0}^{\prime 2}+1\right)-\frac{|\varepsilon|}{\left|\varepsilon^{\prime}\right|}\left(1+2 \ln 0,89 y_{0}^{\prime}\right)\right]} \\
\alpha \simeq \frac{\varphi^{\prime} \mu_{0}\left|\varepsilon^{\prime}\right| \omega^{2}}{2 \gamma_{0}}+\frac{1}{\gamma_{0} a^{\prime 2}} \frac{()}{\left[2 \ln \frac{a^{\prime}}{a}\left(\frac{F}{2} x_{0}^{\prime 2}+1\right)-\frac{|\varepsilon|}{\left|\varepsilon^{\prime}\right|}\left(1+2 \ln 0,89 y_{0}^{\prime}\right)\right]}
\end{gathered}
$$


où

$$
\begin{aligned}
& \text { où } \quad \begin{aligned}
x_{0}^{\prime} \ln \frac{a^{\prime}}{a}\left(\frac{G}{2} x_{0}^{\prime}+\frac{1}{x_{0}^{\prime}} \frac{a^{\prime}}{a} \frac{1}{\left.\ln \frac{a^{\prime}}{a}\right)}|\varepsilon| \omega a^{\prime} R_{\mathrm{s}}+\right. \\
\quad+\left(\varphi|\varepsilon|-\varphi^{\prime}\left|\varepsilon^{\prime}\right|\right) \mu_{0} \omega^{2} a^{\prime 2} \ln \frac{a^{\prime}}{a}\left(\frac{F}{2} x_{0}^{\prime 2}+1\right)+\left(\varphi-\varphi^{\prime}\right) y_{0}^{\prime 2} \frac{|\varepsilon|}{\left|\varepsilon^{\prime}\right|} \ln 0,89 y_{0}^{\prime}
\end{aligned}
\end{aligned}
$$

et $x_{0}^{\prime}$ et $y_{0}^{\prime}$ étant les valeurs de $x^{\prime}$ et $y^{\prime}$ en l'absence de pertes. Dans le cas particulier très important en pratique où le deuxième diélectrique $\left(\varepsilon^{\prime}\right)$ est constitué par de l'air, on a $\varphi^{\prime}=0, \varepsilon^{\prime}=\varepsilon_{0}$ et les expressions (11) et (12) prennent une forme plus simple :

$$
\begin{aligned}
& \delta \gamma_{0} \simeq \frac{1}{\gamma_{0} a^{\prime 2}} \frac{\left[| \varepsilon | \omega a ^ { \prime } x _ { 0 } ^ { \prime } \operatorname { l n } \frac { a ^ { \prime } } { a } \left(\frac{G}{2} x_{0}^{\prime}+\frac{1}{x_{0}^{\prime}} \frac{a^{\prime}}{a} \frac{1}{\left.\left.\ln \frac{a^{\prime}}{a}\right) X_{\mathrm{s}}\right]}\right.\right.}{\left[2 \ln \frac{a^{\prime}}{a}\left(\frac{F}{2} x_{0}^{\prime 2}+1\right)-\frac{|\varepsilon|}{\varepsilon_{0}}\left(1+2 \ln 0,89 y_{0}^{\prime}\right)\right]} \\
& \alpha \simeq \frac{1}{\gamma_{0} a^{\prime 2}}\left[|\varepsilon| \omega a^{\prime} x_{0}^{\prime} \ln \frac{a^{\prime}}{a}\left(\frac{G}{2} x_{0}^{\prime}+\frac{1}{x_{0}^{\prime}} \frac{a^{\prime}}{a} \frac{1}{\ln \frac{a^{\prime}}{a}}\right) R_{\mathrm{s}}\right] \mid(\quad)+ \\
& +\frac{\varphi}{\gamma_{0} a^{\prime 2}}\left[\mu_{0}|\varepsilon| \omega^{2} a^{\prime 2} \ln \frac{a^{\prime}}{a}\left(\frac{F}{2} x_{0}^{\prime 2}+1\right)+y_{0}^{\prime 2} \frac{|\varepsilon|}{\varepsilon_{0}} \ln 0,89 y_{0}^{\prime}\right] /[\text { ] }
\end{aligned}
$$

avec

$$
[\quad]=\left[2 \ln \frac{a^{\prime}}{a}\left(\frac{F}{2} x_{0}^{\prime 2}+1\right)-\frac{|\varepsilon|}{\varepsilon_{0}}\left(1+2 \ln 0,89 y_{0}^{\prime}\right)\right] .
$$

Si de plus $y_{0}^{\prime 2} \ll x_{0}^{\prime 2} \ll 1$

soit

$$
\begin{aligned}
& \delta \gamma_{0} \simeq \frac{\varepsilon_{0} \omega X_{\mathrm{s}}}{2 \gamma_{0} a\left[\frac{\varepsilon_{0}}{|\varepsilon|} \ln \frac{a^{\prime}}{a}-\frac{1}{2}-\ln 0,89 y_{0}^{\prime}\right]} \\
& \alpha \simeq \frac{\varepsilon_{0} \omega R_{\mathrm{s}}}{2 \gamma_{0} a\left[\frac{\varepsilon_{0}}{|\varepsilon|} \ln \frac{a^{\prime}}{a}-\frac{1}{2}-\ln 0,89 y_{0}^{\prime}\right]}-\frac{\varphi \frac{\varepsilon_{0}}{|\varepsilon|-\varepsilon_{0}} \frac{y_{0}^{\prime 2}}{a^{\prime 2}} \ln 0,89 y_{0}^{\prime}}{2 \gamma_{0}\left[\frac{\varepsilon_{0}}{|\varepsilon|} \ln \frac{a^{\prime}}{a}-\frac{1}{2}-\ln 0,89 y_{0}^{\prime}\right]}
\end{aligned}
$$

le premier terme $\alpha_{c}$ correspondant aux pertes dans le métal et le deuxième $\alpha_{d}$ aux pertes dans le diélectrique entourant le métal.

Les expressions (13) à (16) sont valables pour un métal normal ou supraconducteur.

Dans le cas du métal normal :

$$
\alpha_{c} \simeq \delta \gamma_{0} \simeq \frac{\left(\frac{\varepsilon_{0}}{2} \frac{\omega}{\sigma}\right)^{1 / 2}}{2 a\left[\frac{\varepsilon_{0}}{|\varepsilon|} \ln \frac{a^{\prime}}{a}-\frac{1}{2}-\ln 0,89 y_{0}^{\prime}\right]}
$$

$\alpha_{\mathrm{d}}$ étant toujours donné par le terme proportionnel à $\varphi$ dans (14) et (16).

La valeur de $\alpha_{c}$ (et de $\alpha_{d}$ ) diffère de celle obtenue par Goubau, pour les mêmes conditions, par une méthode moins rigoureuse que la nôtre, par la présence du terme supplémentaire $\frac{\varepsilon_{0}}{|\varepsilon|} \ln \frac{a^{\prime}}{a}$ au dénominateur et par une détermination plus précise de $y_{0}^{\prime}$. Les deux expressions ne sont équivalentes que si $y_{0}^{\prime} \rightarrow 0\left(a^{\prime} \rightarrow a\right)$ car alors $-\ln 0,89 y_{0}^{\prime} \gg \frac{1}{2}, \frac{\varepsilon_{0}}{|\varepsilon|} \ln \frac{a^{\prime}}{a}$ et le terme $\frac{1}{2}$ dans l'expression de Goubau est parfaitement négligeable.

Considérons par exemple un cylindre de cuivre, à température ordinaire, entouré de deux diélectriques de permittivités respectives $|\varepsilon|=2 \varepsilon_{0}$ et $\varepsilon_{0}$ et d'angles de pertes $\varphi \simeq 10^{-3}$ et $\varphi^{\prime}=0$ (air). On étudie les variations 
de $\alpha_{\mathrm{c}}$ (atténuation due au conducteur) et $\alpha_{\mathrm{d}}$ (atténuation associée au diélectrique), en fonction de $a^{\prime} / a$ à fréquence donnée $(f=10 \mathrm{GHz})$ (Fig. 1).

On voit que, dans les conditions de l'étude, $\alpha_{c}$ et $\alpha_{d}$ sont du même ordre de grandeur sauf si $a^{\prime} / a$ est très voisin de l'unité ; la localisation de l'énergie au voisinage du conducteur n'est alors pas très bonne car les champs dans le diélectrique externe sont proportionnels à $H_{0}^{(1)}\left(i \kappa^{\prime} r\right)$ ou $H_{1}^{(1)}\left(i \kappa^{\prime} r\right)$ et décroissent d'autant plus lentement que $\kappa^{\prime}$ est faible. Si l'on n'exige pas une localisation très précise de l'énergie au voisinage immédiat du conducteur, on diminuera très notablement $\alpha_{\mathrm{d}}$ en choisissant $a^{\prime}$ voisin de $a$. On aura alors :

$$
\alpha_{d} \ll \alpha_{c} \text { et } \alpha \simeq \alpha_{c} \quad \text { (Fig. 1). }
$$

On a calculé ensuite le coefficient d'atténuation total $\alpha=\alpha_{c}+\alpha_{d}$ dans l'approximation de Goubau et par notre formulation (14).

La figure 2 (variations de $\alpha$ en fonction de $a^{\prime} / a$ pour $f=10 \mathrm{GHz}$ ) montre des écarts déjà importants pour $a^{\prime} / a$ de l'ordre de 1,5 et on voit que l'approximation de Goubau n'est plus acceptable pour $a^{\prime} / a$ supérieur à 3 , elle donne alors une estimation pessimiste de l'atténuation.

Etudions les différents cas de supraconducteurs [7 et 8]. Pour un alliage de type $\mathrm{Nb}_{3} \mathrm{Sn}$ dont la longueur de cohérence $\xi_{0}$ ainsi que le libre parcours moyen sont faibles par rapport à la profondeur de pénétration des champs, on a :

$$
Z_{\mathrm{s}}=\frac{i \mu_{0} \omega}{\left[\left(\frac{\xi}{\lambda_{\mathrm{L}}^{2} \xi_{0}}\right)^{2}+\left(\mu_{0} \omega \sigma\right)^{2}\right]^{1 / 4}} \exp \left[-\frac{i}{2} \operatorname{Arctg} \mu_{0} \omega \sigma \lambda_{\mathrm{L}}^{2} \frac{\xi_{0}}{\xi}\right]
$$

On obtient alors :

$$
\delta \gamma_{0} \simeq \frac{\sqrt{\mu_{0} \varepsilon_{0}} \omega \cos \left[\frac{1}{2} \operatorname{Arctg} \mu_{0} \omega \sigma \lambda_{\mathrm{L}}^{2}\right]}{2 a\left[\frac{1}{\lambda_{\mathrm{L}}^{4}}+\left(\mu_{0} \omega \sigma\right)^{2}\right]^{1 / 4}\left[\frac{\varepsilon_{0}}{|\varepsilon|} \ln \frac{a^{\prime}}{a}-\frac{1}{2}-\ln 0,89 y_{0}^{\prime}\right]}
$$

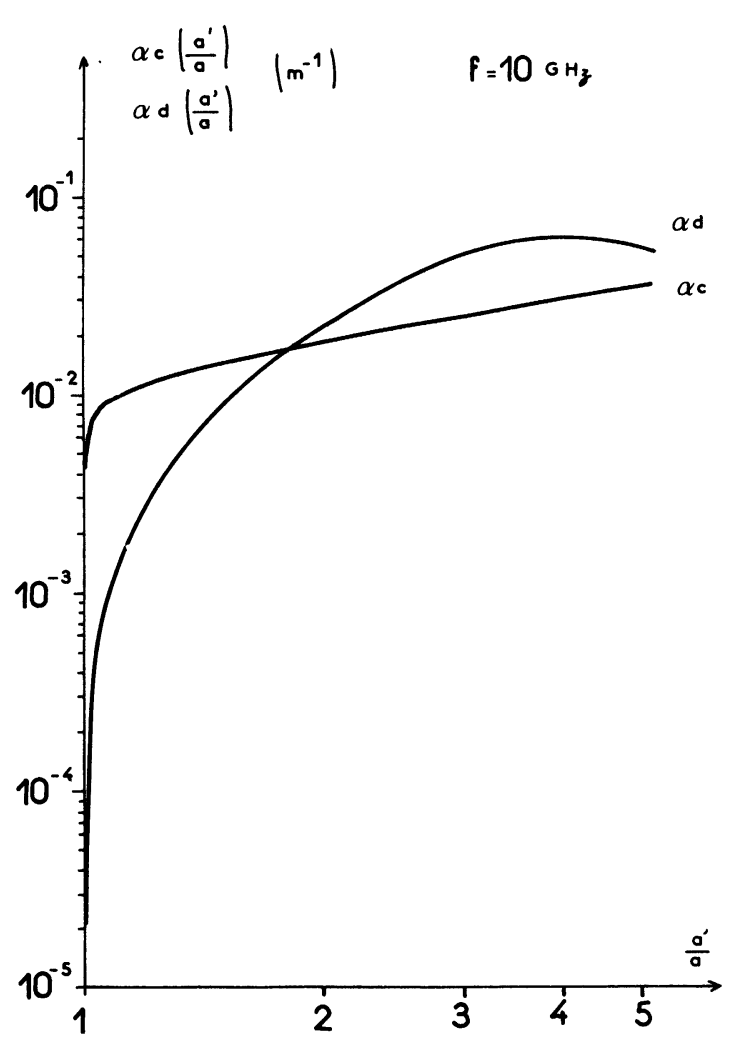

Fig. 1. - Atténuation $\alpha_{c}\left(a^{\prime} / a\right)$ et $\alpha_{d}\left(a^{\prime} / a\right)$ pour $f=10 \mathrm{GHz}$ dans le cas du cuivre.

[Attenuation factor $\alpha_{\mathrm{c}}\left(a^{\prime} / a\right)$ and $\alpha_{\mathrm{d}}\left(a^{\prime} / a\right)$ for copper at $f=10 \mathrm{GHz}$.]

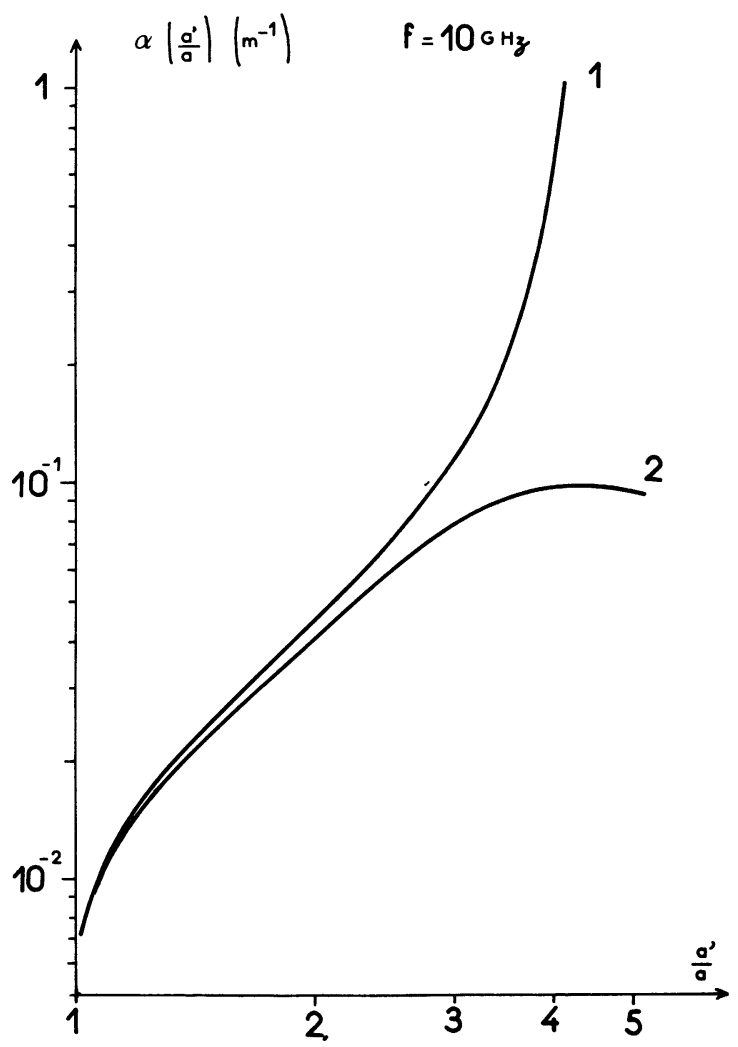

Fig. 2. - Atténuation totale $\alpha\left(a^{\prime} / a\right)$ pour $f=10 \mathrm{GHz}$ : courbe 1 : expression de Goubau ; courbe 2 : expression (14).

[Total attenuation factor $\alpha\left(a^{\prime} / a\right)$ for $f=10 \mathrm{GHz}$ : curve 1 : Goubau's theory ; curve 2 : expression (14).] 


$$
\alpha_{\mathrm{c}} \simeq \frac{\sqrt{\mu_{0} \varepsilon_{0}} \omega \sin \left[\frac{1}{2} \operatorname{Arctg} \mu_{0} \omega \sigma \lambda_{\mathrm{L}}^{2}\right]}{2 a\left[\frac{1}{\lambda_{\mathrm{L}}^{4}}+\left(\mu_{0} \omega \sigma\right)^{2}\right]^{1 / 4}\left[\frac{\varepsilon_{0}}{|\varepsilon|} \ln \frac{a^{\prime}}{a}-\frac{1}{2}-\ln 0,89 y_{0}^{\prime}\right]} .
$$

Pour un métal pur supraconducteur le libre parcours moyen $l$ est grand à basse température. Si de plus la longueur de cohérence $\xi_{0}$ est élevée (cas de l'aluminium par exemple), l'impédance de surface est donnée par :

$$
Z_{\mathrm{s}} \simeq \frac{\sqrt{3}}{2}\left(\frac{4}{3 \pi}\right)^{1 / 3} \frac{i \mu_{0} \omega}{\left[\left(\frac{1}{\xi_{0} \lambda_{\mathrm{L}}^{2}}\right)^{2} \cdot\left(\frac{\mu_{0} \omega \sigma}{l}\right)^{2}\right]^{1 / 6}} \exp \left[-\frac{i}{3} \operatorname{Arctg} \mu_{0} \omega \sigma \lambda_{\mathrm{L}}^{2} \frac{\xi_{0}}{l}\right]
$$

d'où

$$
\begin{aligned}
& \delta \gamma_{0} \simeq \frac{\sqrt{3}}{2}\left(\frac{4}{3 \pi}\right)^{1 / 3} \frac{\sqrt{\mu_{0} \varepsilon_{0}} \omega \cos \left[\frac{1}{3} \operatorname{Arctg} \mu_{0} \omega \sigma \lambda_{\mathrm{L}}^{2} \frac{\xi_{0}}{l}\right]}{2 a\left[\left(\frac{1}{\xi_{0} \lambda_{\mathrm{L}}^{2}}\right)^{2}+\left(\frac{\mu_{0} \omega \sigma}{l}\right)^{2}\right]^{1 / 6}\left[\frac{\varepsilon_{0}}{|\varepsilon|} \ln \frac{a^{\prime}}{a}-\frac{1}{2}-\ln 0,89 y_{0}^{\prime}\right]} \\
& \alpha_{c} \simeq \frac{\sqrt{3}}{2}\left(\frac{4}{3 \pi}\right)^{1 / 3} \frac{\sqrt{\mu_{0} \varepsilon_{0}} \omega \sin \left[\frac{1}{3} \operatorname{Arctg} \mu_{0} \omega \sigma \lambda_{\mathrm{L}}^{2} \frac{\xi_{0}}{l}\right]}{2 a\left[\left(\frac{1}{\xi_{0} \lambda_{\mathrm{L}}^{2}}\right)^{2}+\left(\frac{\mu_{0} \omega \sigma}{l}\right)^{2}\right]^{1 / 6}\left[\frac{\varepsilon_{0}}{|\varepsilon|} \ln \frac{a^{\prime}}{a}-\frac{1}{2}-\ln 0,89 y_{0}^{\prime}\right]}
\end{aligned}
$$

avec toujours :

$$
\alpha_{\mathrm{d}} \simeq-\frac{\frac{\varphi \varepsilon_{0}}{|\varepsilon|-\varepsilon_{0}} \frac{y_{0}^{\prime 2}}{a^{\prime 2}} \ln 0,89 y_{0}^{\prime}}{2 \gamma_{0}\left[\frac{\varepsilon_{0}}{|\varepsilon|} \ln \frac{a^{\prime}}{a}-\frac{1}{2}-\ln 0,89 y_{0}^{\prime}\right]} .
$$

Les formules (21) et (24) permettent de calculer $\alpha_{c}$ pour tous les types de supraconducteurs.

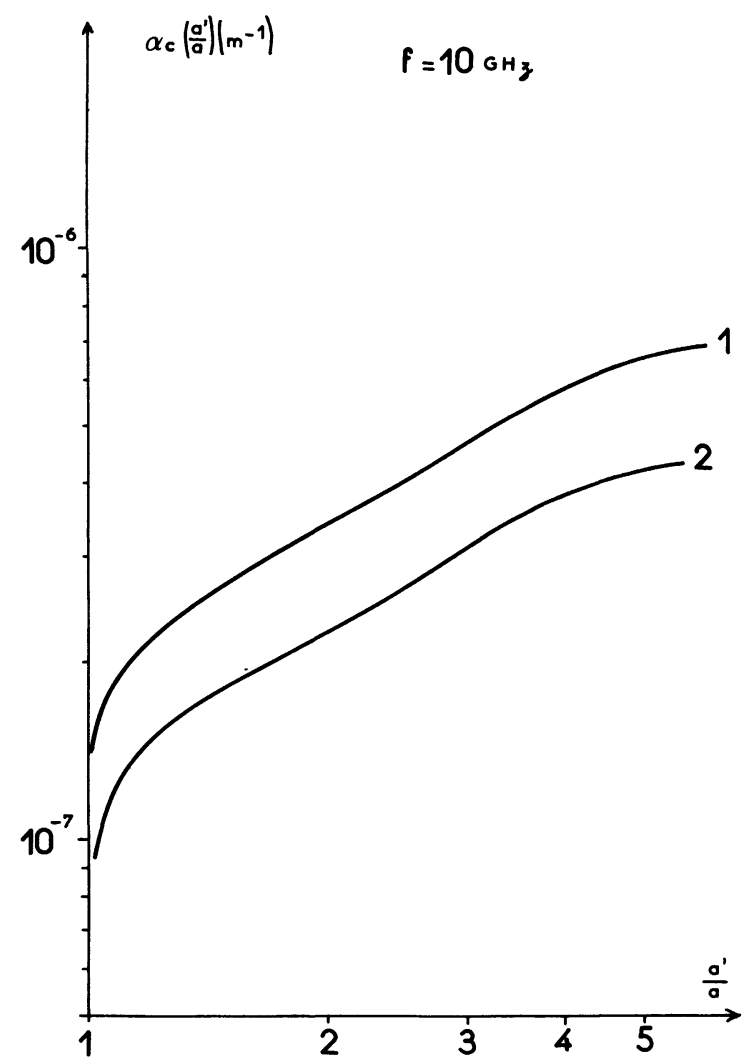

Fig. 3. - Atténuation $\alpha_{\mathrm{c}}\left(a^{\prime} / a\right)$ dans le cas des supraconducteurs pour $f=10 \mathrm{GHz}$ : courbe 1 : alliage $\mathrm{Nb}_{3} \mathrm{Sn}$, expression (14); courbe 2 : aluminium, expression (14).

[Attenuation factor $\alpha_{\mathrm{c}}\left(a^{\prime} / a\right)$ for superconductors at $f=10 \mathrm{GHz}$ : curve $1: \mathrm{Nb}_{3} \mathrm{Sn}$ alloy, expression (14); curve 2 : aluminum, expression (14).] 
La figure 3 représente $\alpha_{\mathrm{c}}\left(a^{\prime} / a\right)$ pour $\mathrm{Nb}_{3} \operatorname{Sn}\left(\xi_{0} \simeq 50 \AA\right)$ et $\mathrm{Al}\left(\xi_{0} \simeq 16000 \AA\right)$ aux températures $T=T_{\mathrm{c}} / 4$.

Comme il était prévisible, l'atténuation due aux métaux supraconducteurs est très faible et peut être rendue plus faible que celle correspondant à un guide supraconducteur cylindrique creux [9] si $a^{\prime}$ est assez voisin de $a$.

Il convient de remarquer que le terme prépondérant de l'atténuation totale $\alpha=\alpha_{c}+\alpha_{d}$ est $\alpha_{d}$ qui ne peut être rendu très inférieur à $\alpha_{c}$ comme dans le cas d'un métal ordinaire.

Notons cependant que la valeur de $\alpha_{d}$ aux très basses températures est très nettement inférieure à celle considérée à la température ambiante.

Il semblerait donc que l'utilisation d'un diélectrique à très faible angle de perte permette d'envisager une atténuation globale comparable à celle du guide cylindrique classique supraconducteur.

Conclusion. - L'introduction de la notion d'impédance de surface dans l'étude de la propagation d'une onde électromagnétique de surface nous a permis d'écrire des expressions analytiques des coefficients d'atténuation et du déphasage plus précises que celles jusqu'alors utilisées.

De plus nous avons pu traiter les cas très complexes des matériaux supraconducteurs, le cas du métal ordinaire n'apparaissant alors que comme un cas particulier de notre théorie. L'exploitation numérique de nos résultats analytiques, dans le cas d'un métål normal, montre que l'expression du coefficient d'atténuation donnée par Goubau et reprise par de nombreux auteurs n'est acceptable que pour une couche très mince de diélectrique. Notre formulation donne des résultats utilisables pour des couches diélectriques dont l'épaisseur peut aller jusqu'à 5 fois le rayon du conducteur; ceci favorise la concentration de l'énergie au voisinage du conducteur tout en gardant au coefficient d'atténuation une valeur faible. Des études expérimentales sont en cours.

\section{Bibliographie}

[1] Goubau, G., J. Appl. Phys. 21 (1950) 1119.

[2] Berceli, T., Acta Tech. Acad. Sci. Hung. 25 (1959) 257.

[3] Severin, H., Rev. Tech. Philipps 26 (1965) 114.

[4] Huey, R. W., Cheong, K. L., Instr. Radioelectron. Austral 26 (1965) 301.
[5] Severin, H., Tech. Mitt. P.T.T. 9 (1968) 442.

[6] CHIBA, J., IEEE Trans. Microwave 25 (1977) 94.

[7] ZEPP, G., J. Physique 31 (1970) 513.

[8] Zepp, G., Revue Phys. Appl. 6 (1971) 135.

[9] Zepp, G., Wick, A., Fabre, G., Can. J. Phys. 55 (1977) 1551 